\title{
On the product in negative Tate cohomology for finite groups
}

\author{
HAgGai TENE
}

Our aim in this paper is to give a geometric description of the cup product in negative degrees of Tate cohomology of a finite group with integral coefficients. By duality it corresponds to a product in the integral homology of $B G$ :

$$
H_{n}(B G, \mathbb{Z}) \otimes H_{m}(B G, \mathbb{Z}) \rightarrow H_{n+m+1}(B G, \mathbb{Z})
$$

for $n, m>0$. We describe this product as join of cycles, which explains the shift in dimensions. Our motivation came from the product defined by Kreck using stratifold homology. We then prove that for finite groups the cup product in negative Tate cohomology and the Kreck product coincide. The Kreck product also applies to the case where $G$ is a compact Lie group (with an additional dimension shift).

20J06, 55R40

\section{Introduction}

For a finite group $G$ one defines Tate cohomology with coefficients in a $\mathbb{Z}[G]$ module $M$, denoted by $\hat{H}^{*}(G, M)$. This is a multiplicative theory:

$$
\widehat{H}^{n}(G, M) \otimes \widehat{H}^{m}\left(G, M^{\prime}\right) \rightarrow \widehat{H}^{n+m}\left(G, M \otimes M^{\prime}\right)
$$

and the product is called cup product. For $n>0$ there is a natural isomorphism $H^{n}(G, M) \rightarrow \hat{H}^{n}(G, M)$, and for $n<-1$ there is a natural isomorphism $\hat{H}^{n}(G, M) \rightarrow$ $H_{-n-1}(G, M)$. We restrict ourselves to coefficients in the trivial module $\mathbb{Z}$. In this case, $\hat{H}^{*}(G, \mathbb{Z})$ is a graded ring. Also, in this case the group cohomology and homology are actually the cohomology and homology of a topological space, namely $B G$, the classifying space of principal $G$ bundles: $H^{n}(G, \mathbb{Z}) \cong H^{n}(B G, \mathbb{Z})$ and $H_{n}(G, \mathbb{Z}) \cong H_{n}(B G, \mathbb{Z})$. Combining this with the isomorphism we had before $\hat{H}^{n}(G, \mathbb{Z}) \rightarrow H_{-n-1}(G, \mathbb{Z})$ for $n<-1$ we get a product $H_{n}(B G, \mathbb{Z}) \otimes H_{m}(B G, \mathbb{Z}) \rightarrow$ $H_{n+m+1}(B G, \mathbb{Z})$ for $n, m>0$. Note the dimension shift. This product, with coefficients in a field of characteristics $p$ rather than $\mathbb{Z}$, was studied by Benson and Carlson [1]. Our aim in this paper is to give a geometric description of this product. We give a rather concrete description in singular homology that involves the join of cycles, and that explains the shift in dimension. 
Our motivation came from a geometric description of $H_{*}(G, \mathbb{Z})$ given by Kreck in [6] and the product he defined using stratifold homology. We then prove that the cup product in negative Tate cohomology and the Kreck product coincide. An advantage in Kreck theory is that it holds also for compact Lie groups giving a product:

$$
H_{n}(B G, \mathbb{Z}) \otimes H_{m}(B G, \mathbb{Z}) \rightarrow H_{n+m+1+\operatorname{dim}(G)}(B G, \mathbb{Z}) .
$$

Acknowledgements This paper is a part of the author's $\mathrm{PhD}$ thesis, written under the direction of Professor Matthias Kreck in the Hausdorff Research Institute for Mathematics (HIM). The author would like to thank Professor Kreck for his support, the HIM for the time he spent there, and the HCM for their financial support.

\section{Tate cohomology}

Remark In this paper $R$ is assumed to be a ring with unit, not necessarily commutative, and all modules are assumed to be left $R$-modules unless stated otherwise. The group $G$ is assumed to be finite unless stated otherwise.

We start by defining Tate cohomology and the cup product as appears in Carlson et al [3]. To do so we introduce the language taken from the stable module category. We will not get into details, for a formal treatment the reader is referred to the appendix.

Let $M, N$ be two $R$-modules, denote by $\underline{\operatorname{Hom}}_{R}(M, N)$ the quotient of $\operatorname{Hom}_{R}(M, N)$ by the maps that factor through some projective module.

Definition 2.1 Given an $R$-module $M$, denote by $\Omega^{k} M$ the following module:

Take any partial projective resolution of $M$,

$$
P_{k-1} \stackrel{d_{k-1}}{\longrightarrow} P_{k-2} \rightarrow \cdots \rightarrow P_{1} \rightarrow P_{0} \rightarrow M
$$

then $\Omega^{k} M=\operatorname{ker}\left(d_{k-1}: P_{k-1} \rightarrow P_{k-2}\right)$. If $k=1$ then we simply denote it by $\Omega M$. This module clearly depends on the choice of the resolution. Nevertheless, as proved in the appendix, the modules $\underline{\operatorname{Hom}}_{R}\left(\Omega^{k} M, \Omega^{l} N\right)$ do not depend on the choice of resolutions, ie, they are well defined up to canonical isomorphisms. If we would like to stress the dependency on $P$ then we would use the notation $\Omega_{P}^{k} M$.

Note that there is a natural map $\Psi: \underline{\operatorname{Hom}}_{R}(M, N) \rightarrow \underline{\operatorname{Hom}}_{R}(\Omega M, \Omega N)$. 
Definition 2.2 The Tate cohomology of $G$ with coefficients in a $\mathbb{Z}[G]$ module $M$ is given by

$$
\widehat{H}^{n}(G, M)=\widehat{\operatorname{Ext}}_{\mathbb{Z}[G]}^{n}(\mathbb{Z}, M)=\underset{m}{\lim } \underline{\operatorname{Hom}}_{\mathbb{Z}[G]}\left(\Omega^{n+m} \mathbb{Z}, \Omega^{m} M\right)
$$

where $\mathbb{Z}$ is the trivial $\mathbb{Z}[G]$-module (if $n<0$ then we start this sequence from $m=-n$ ).

In our case, where $G$ is finite, we have the following proposition which is proved in the appendix:

Proposition 2.3 If $G$ is a finite group and $M$ is a $\mathbb{Z}[G]$-module which is projective as a $\mathbb{Z}$-module then the homomorphism $\Psi: \underline{\operatorname{Hom}}_{\mathbb{Z}[G]}(M, N) \rightarrow \underline{\operatorname{Hom}}_{\mathbb{Z}[G]}(\Omega M, \Omega N)$ is an isomorphism.

Therefore, since $\mathbb{Z}$ and $\Omega^{k} \mathbb{Z}$ are projective as $\mathbb{Z}$-modules this limit equals

$$
\hat{H}^{n}(G, M)=\underline{\operatorname{Hom}}_{\mathbb{Z}[G]}\left(\Omega^{n} \mathbb{Z}, M\right)
$$

if $n \geq 0$ or

$$
\widehat{H}^{n}(G, M)=\underline{\operatorname{Hom}}_{\mathbb{Z}[G]}\left(\mathbb{Z}, \Omega^{-n} M\right)
$$

if $n<0$. Our main interest will be the second case, especially when $M=\mathbb{Z}$.

Example 2.4 $\hat{H}^{-1}(G, \mathbb{Z})=\underline{\operatorname{Hom}}_{\mathbb{Z}[G]}(\mathbb{Z}, \Omega \mathbb{Z})$. Take the exact sequence

$$
0 \rightarrow I \rightarrow \mathbb{Z}[G] \stackrel{f}{\rightarrow} \mathbb{Z} \rightarrow 0,
$$

where the map $f$ is the augmentation map and $I$ is the augmentation ideal, so $I=\Omega \mathbb{Z}$. Therefore $\operatorname{Hom}_{\mathbb{Z}[G]}(\mathbb{Z}, \Omega \mathbb{Z}) \cong \operatorname{Hom}_{\mathbb{Z}[G]}(\mathbb{Z}, I)=\{0\}$ so $\hat{H}^{-1}(G, \mathbb{Z})=\{0\}$.

Let $G$ be a finite group. We construct a natural isomorphism $\hat{H}^{-n-1}(G, \mathbb{Z}) \rightarrow$ $H_{n}(G, \mathbb{Z})$ for $n \geq 1$. Before that we prove a small lemma.

Lemma 2.5 Let $G$ be a finite group and $P$ a projective $\mathbb{Z}[G]-$ module, then for every element $x \in P$ we have:

(1) $x \in P^{G} \Leftrightarrow \exists y \in P, x=N y$,

(2) $y \otimes 1=y^{\prime} \otimes 1 \in P \otimes_{\mathbb{Z}[G]} \mathbb{Z} \Leftrightarrow N y=N y^{\prime}$,

where $P^{G}$ are the invariants of $P$ under the action of $G, N$ is the norm homomorphism defined by multiplication by the element $N=\sum_{g \in G} g \in \mathbb{Z}[G]$. 
Proof For every $\mathbb{Z}[G]$-module $M$ the following sequence is exact:

$$
0 \rightarrow \hat{H}^{-1}(G, M) \rightarrow H_{0}(G, M) \rightarrow H^{0}(G, M) \rightarrow \hat{H}^{0}(G, M) \rightarrow 0,
$$

where the map $H_{0}(G, M) \rightarrow H^{0}(G, M)$ is the norm map $N: M \otimes \mathbb{Z} \rightarrow M^{G}$ given by $(N(x \otimes k)=k N x)$ (see Brown [2, VI,4]). If $M$ is projective then $\hat{H}^{m}(G, M)=0$ for all $m \in \mathbb{Z}$, hence $N$ is an isomorphism. We conclude:

(1) Surjectivity of $N$ implies that $x \in P^{G} \Leftrightarrow \exists y \in P, x=N y$.

(2) Injectivity of $N$ implies that $y \otimes 1=y^{\prime} \otimes 1 \Leftrightarrow N y=N y^{\prime}$ for all $y, y^{\prime} \in P$.

Proposition 2.6 Let $G$ be a finite group then there is an isomorphism between $\widehat{H}^{-n-1}(G, \mathbb{Z})$ and $H_{n}(G, \mathbb{Z})$ for $n \geq 1$.

Proof Take a projective resolution of $\mathbb{Z}$

$$
\cdots \rightarrow P_{n} \stackrel{d_{n}}{\rightarrow} P_{n-1} \cdots \rightarrow P_{0} \rightarrow \mathbb{Z} .
$$

Taking the tensor of it with $\mathbb{Z}$ gives us the chain complex for the homology of $G$ which we denote by $C_{*}(G)$. We define a map $\Phi$ from $\operatorname{Hom}_{\mathbb{Z}[G]}\left(\mathbb{Z}, \Omega^{n+1} \mathbb{Z}\right)$ to $C_{n}(G)$ the following way: Given a homomorphism $f: \mathbb{Z} \rightarrow \Omega^{n+1} \mathbb{Z}, f(1)=x$ is an invariant element in $P_{n}$. By the lemma, since $P_{n}$ is projective and $x$ is invariant, there is some $y \in P_{n}$ such that $x=N y$. We define $\Phi(f)=y \otimes 1$. This doesn't depend on the choice of $y$ since $N y=N y^{\prime} \Leftrightarrow y \otimes 1=y^{\prime} \otimes 1$ by the lemma above. We know that $\operatorname{Nd}_{n}(y)=d_{n}(N y)=d_{n}(x)=0$ and by the lemma this implies that $d_{n}(y) \otimes 1=0$ ( $P_{n-1}$ is projective and here we use the fact that $n \geq 1$ ). We deduce that $y \otimes 1 \in Z_{n}(G)$. The map described now $\operatorname{Hom}_{\mathbb{Z}[G]}\left(\mathbb{Z}, \Omega^{n+1} \mathbb{Z}\right) \rightarrow Z_{n}(G)$ is surjective since given an element $y \otimes 1 \in C_{n}(G)$ such that $d_{n}(y) \otimes 1=0$ this implies that $\operatorname{Nd}_{n}(y)=0$, so we define $f(k)=k N y$, this is well defined since $N y$ is invariant and in the kernel of $d_{n}$.

We now have a surjective homomorphism $\Phi: \operatorname{Hom}_{\mathbb{Z}[G]}\left(\mathbb{Z}, \Omega^{n+1} \mathbb{Z}\right) \rightarrow H_{n}(G, \mathbb{Z})$. If $f \in \operatorname{ker}(\Phi)$ then there exist $s \in P_{n+1}$ such that $\Phi(f)=y \otimes 1=d_{n+1}(s) \otimes 1$. This implies that the map $f: \mathbb{Z} \rightarrow \Omega^{n+1} \mathbb{Z}$ factors as a $\mathbb{Z}[G]$ map through $P_{n+1}$, which is projective, by $1 \mapsto N s$. On the other hand if $f$ factors through a projective module, without loss of generality, $P_{n+1}$, then $N y=f(1)=d_{n+1}(N s)$ (every invariant element in $P_{n+1}$ is of the form $N s$ by the lemma). This implies that $\operatorname{Nd}_{n+1}(s)=N y$ if and only if $d_{n+1}(s \otimes 1)=d_{n+1}(s) \otimes 1=y \otimes 1$.

We conclude that the induced map

$$
\underline{\Phi:} \hat{H}^{-n-1}(G, \mathbb{Z})=\underline{\operatorname{Hom}}_{\mathbb{Z}[G]}\left(\mathbb{Z}, \Omega^{n+1} \mathbb{Z}\right) \rightarrow H_{n}(G, \mathbb{Z})
$$

is an isomorphism for all $n \geq 1$. 
Remark Since $\hat{H}^{-1}(G, \mathbb{Z})=\{0\}$ we conclude that $\hat{H}^{-n-1}(G, \mathbb{Z}) \cong \widetilde{H}_{n}(G, \mathbb{Z})$ for $n \geq 0$ where $\widetilde{H}_{n}(G, \mathbb{Z})$ is the reduced homology.

\section{The product structure}

The cup product in Tate cohomology $\hat{H}^{-n}(G, \mathbb{Z}) \otimes \hat{H}^{-m}(G, \mathbb{Z}) \rightarrow \hat{H}^{-n-m}(G, \mathbb{Z})$ is given by composition (this is also called the Yoneda composition product). Given

$$
\begin{aligned}
& {[f] \in \hat{H}^{-n}(G, \mathbb{Z})=\underline{\operatorname{Hom}}_{\mathbb{Z}[G]}\left(\mathbb{Z}, \Omega^{n} \mathbb{Z}\right),} \\
& {[g] \in \hat{H}^{-m}(G, \mathbb{Z})=\underline{\operatorname{Hom}}_{\mathbb{Z}[G]}\left(\mathbb{Z}, \Omega^{m} \mathbb{Z}\right) \cong \underline{\operatorname{Hom}}_{\mathbb{Z}[G]}\left(\Omega^{n} \mathbb{Z}, \Omega^{n+m} \mathbb{Z}\right),}
\end{aligned}
$$

we compose them to get a map

$$
[f] \cup[g]=[g \circ f] \in \underline{\operatorname{Hom}}_{\mathbb{Z}[G]}\left(\mathbb{Z}, \Omega^{n+m} \mathbb{Z}\right) .
$$

Since for $n, m \geq 2$ we have $\hat{H}^{-n}(G, \mathbb{Z}) \cong H_{n-1}(G, \mathbb{Z}), \hat{H}^{-m}(G, \mathbb{Z}) \cong H_{m-1}(G, \mathbb{Z})$ we have a product $H_{n-1}(G, \mathbb{Z}) \otimes H_{m-1}(G, \mathbb{Z}) \rightarrow H_{n+m-1}(G, \mathbb{Z})$. We would like to have a description of the isomorphism $\underline{\operatorname{Hom}}_{\mathbb{Z}[G]}\left(\mathbb{Z}, \Omega^{m} \mathbb{Z}\right) \cong \underline{\operatorname{Hom}}_{\mathbb{Z}[G]}\left(\Omega^{n} \mathbb{Z}, \Omega^{n+m} \mathbb{Z}\right)$ which is concrete. To do so we use the following construction.

\section{The join of augmented chain complexes}

Let $G$ be a finite group and let $P$ and $Q$ be the following augmented chain complexes over $\mathbb{Z}[G]: \cdots \rightarrow P_{2} \rightarrow P_{1} \rightarrow P_{0} \rightarrow \mathbb{Z}$ and $\cdots \rightarrow Q_{2} \rightarrow Q_{1} \rightarrow Q_{0} \rightarrow \mathbb{Z}$. We define the join of those two chain complexes to be $P * Q=\Sigma\left(P \otimes_{\mathbb{Z}} Q\right)$ that is the suspension of the tensor product over $\mathbb{Z}$ (with a diagonal $G$ action). To be more specific $(P * Q)_{n}=\bigoplus_{0 \leq k \leq n+1} P_{k-1} \otimes_{\mathbb{Z}} Q_{n-k}$ :

$$
\cdots \rightarrow P_{1} \otimes_{\mathbb{Z}} \mathbb{Z} \oplus P_{0} \otimes_{\mathbb{Z}} Q_{0} \oplus \mathbb{Z} \otimes_{\mathbb{Z}} Q_{1} \rightarrow P_{0} \otimes_{\mathbb{Z}} \mathbb{Z} \oplus \mathbb{Z} \otimes_{\mathbb{Z}} Q_{0} \rightarrow \mathbb{Z} \otimes_{\mathbb{Z}} \mathbb{Z} .
$$

$P * Q$ is an augmented $\mathbb{Z}[G]$ chain complex in a natural way.

Lemma 3.1 If both $P$ and $Q$ are projective and acyclic augmented $\mathbb{Z}[G]$ chain complexes then $P * Q$ is a projective and acyclic augmented $\mathbb{Z}[G]$ chain complex.

Proof $P$ and $Q$ are projective acyclic chain complexes over $\mathbb{Z}$ so the same is true for their tensor product, by the Künneth formula. $(P * Q)_{n}$ is projective over $\mathbb{Z}[G]$ for $n \geq 0$ since each of the modules $P_{k-1} \otimes_{\mathbb{Z}} Q_{n-k}$ is projective.

Lemma 3.2 Let $P$ and $Q$ be two resolutions of $\mathbb{Z}$ over $\mathbb{Z}[G]$, and let $s \in Q_{n-1}$ be an element, $n>1$. Define a map $s_{*}: P_{k-1} \rightarrow(P * Q)_{k+n-1}$ by $s_{*}(x)=x \otimes s$ called the join with $s$. Then we have: 
(1) $S_{*}$ is a group homomorphism.

(2) If $s$ is $G$-invariant then $s_{*}$ is a homomorphism over $\mathbb{Z}[G]$.

(3) If $s \in \operatorname{ker}\left(Q_{n-1} \rightarrow Q_{n-2}\right)$ then $s_{*}$ is a chain map of degree $n$.

Proof (1) This follows from the properties of the tensor product.

(2) For every $g \in G$ we have $g\left(s_{*}(x)\right)=g(x \otimes s)=g(x) \otimes g(s)=g(x) \otimes s=s_{*}(g(x))$.

(3) $\partial\left(s_{*}(x)\right)=\partial(x \otimes s)=\partial(x) \otimes s+(-1)^{|x|+1} x \otimes \partial s=\partial(x) \otimes s=s_{*}(\partial(x))$.

This implies the following:

Theorem 3.3 Let $n, m>0$. Then the product

$$
\hat{H}^{-n}(G, \mathbb{Z}) \otimes \hat{H}^{-m}(G, \mathbb{Z}) \rightarrow \hat{H}^{-n-m}(G, \mathbb{Z})
$$

is given by $[f] \cup[g]=[f * g]$ where $(f * g)(k)=k \cdot f(1) \otimes g(1) \in \Omega_{P * P}^{m+n} \mathbb{Z}$ for all $k \in \mathbb{Z}$.

Proof Take a projective resolution $P$ for $\mathbb{Z}$ over $\mathbb{Z}[G]$. Let

$$
\begin{aligned}
& {[f] \in \hat{H}^{-n}(G, \mathbb{Z})=\underline{\operatorname{Hom}}_{\mathbb{Z}[G]}\left(\mathbb{Z}, \Omega^{n} \mathbb{Z}\right),} \\
& {[g] \in \hat{H}^{-m}(G, \mathbb{Z})=\underline{\operatorname{Hom}}_{\mathbb{Z}[G]}\left(\mathbb{Z}, \Omega^{m} \mathbb{Z}\right) \cong \underline{\operatorname{Hom}}_{\mathbb{Z}[G]}\left(\Omega^{n} \mathbb{Z}, \Omega^{n+m} \mathbb{Z}\right) .}
\end{aligned}
$$

Choose representatives $f, g$ and define a degree $m$ map $P \rightarrow P * P$ by $x \mapsto x \otimes g(1)$. Since $g(1)$ is invariant and in the kernel of $d_{m-1}$, this map is a chain map of $\mathbb{Z}[G]$ chain complexes of degree $m$. This gives us a concrete construction of the isomorphism $\underline{\operatorname{Hom}}_{\mathbb{Z}[G]}\left(\mathbb{Z}, \Omega^{m} \mathbb{Z}\right) \cong \underline{\operatorname{Hom}}_{\mathbb{Z}[G]}\left(\Omega^{n} \mathbb{Z}, \Omega^{n+m} \mathbb{Z}\right)$. The composition is therefore $g \circ$ $f(1)=f(1) \otimes g(1)$.

\section{A description of the product by joins of cycles}

We now consider resolutions which come from singular chains of spaces. Let $G$ be a finite group. Recall that a contractible $G-\mathrm{CW}$-complex with a free $G$ action is denoted by $E G$, the quotient space $E G / G$ is the classifying space of principal $G$ bundles and is denoted by $B G$.

We consider now the augmented singular chain complex of $E G$ denoted by $C_{*}(E G)$. The action on $E G$ is free so $C_{*}(E G)$ is projective $(n \geq 0)$ and $E G$ is contractible so $C_{*}(E G)$ is acyclic.

As we saw before in Proposition 2.6 every element of $H_{n}(G, \mathbb{Z})$ can be considered as an invariant cycle in $C_{n}(E G)$ (modulo invariant boundary). We will show that the 
product can be considered as the join of the two such cycles, which is naturally an invariant cycle in $C_{*}(E G * E G)$ where $E G * E G$ is the join of two copies of $E G$. Note that since the join of contractible spaces is contractible, $E G * E G$ is contractible, and it has a natural $G$ action, given by $g(x, y, t)=(g x, g y, t)$, which is free since it is free on both copies of $E G$. This implies that its augmented singular chain complex is a projective resolution of $\mathbb{Z}$ over $\mathbb{Z}[G]$.

We now associate the join of chain complexes to the join of spaces.

Lemma 3.4 Let $A$ and $B$ be two spaces and let $C_{*}(A)$ and $C_{*}(B)$ be their augmented singular chain complexes, then there is a natural chain map

$$
h: C_{*}(A) * C_{*}(B) \rightarrow C_{*}(A * B) .
$$

If $G$ acts on $A$ and $B$ then it also acts on $A * B$ and the chain complexes are complexes over $\mathbb{Z}[G]$ and $h$ is a map of $\mathbb{Z}[G]$ chain complexes.

Proof We first note that for $n, m \geq 0$, for every two singular simplices $\sigma \in C_{n}(A)$ and $\tau \in C_{m}(B)$ there is a canonical singular chain $\sigma * \tau \in C_{n+m+1}(A * B)$ and this definition can be extended in a bilinear way to chains. Define $h$ the following way:

Given an element $s \otimes t \in C_{n}(A) \otimes C_{m}(B)$, if $n, m \geq 0$ then $h(s \otimes t)=s * t$, else $n=-1$ (or $m=-1$ ) then $s$ is an integer, denote it by $k$ then $h(s \otimes t)=h(k \otimes t)=k \cdot t$ where $t$ is the chain induced by the inclusion of $B$ in $A * B$ (and similarly for $m=-1$ ).

We have to show that $h$ is a chain map. For two simplices of positive dimension we have the formula $\partial(\sigma * \tau)=\partial(\sigma) * \tau+(-1)^{\operatorname{dim}(\sigma)+1} \sigma * \partial(\tau)$. The formula extends to chains, so we have

$$
\begin{aligned}
\partial h(s \otimes t)=\partial(s * t) & =\partial(s) * t+(-1)^{|s|+1} s * \partial(t) \\
& =h\left(\partial(s) \otimes t+(-1)^{|s|+1} s \otimes \partial(t)\right)=h(\partial(s \otimes t)) .
\end{aligned}
$$

For $\sigma$, a simplex of dimension 0 (a point), $\sigma * \tau$ is the cone over $\tau$ and its boundary is given by $\partial(\sigma * \tau)=\tau+(-1)^{\operatorname{dim}(\sigma)+1} \sigma * \partial(\tau)$. Since the boundary map $C_{0}(A) \rightarrow \mathbb{Z}$ is the augmentation map we see indeed that also in this case $h$ commutes with the boundary (with respect to the way we have defined $h(k \otimes t)$ ).

The boundary formula is not true when one of the simplices is zero dimensional due to the nonsymmetric way we define the faces of a zero simplex (the $n$ simplex has $n+1$ faces while the zero simplex has no faces). If we wanted to be consistent with the boundaries of the higher simplices then we should have used only augmented chain complexes. More in this direction appears in [4]. 
When there is a $G$ action on both spaces then clearly all the complexes are complexes over $\mathbb{Z}[G] . h$ is a $\mathbb{Z}[G]$ chain map since for every $g \in G$ we have

$$
h(g(s \otimes t))=h(g s \otimes g t))=g s * g t=g(s * t)=g(h(s \otimes t)) .
$$

Theorem 3.5 The cup product in negative Tate cohomology gives a product

$$
H_{n}(G, \mathbb{Z}) \otimes H_{m}(G, \mathbb{Z}) \rightarrow H_{n+m+1}(G, \mathbb{Z})
$$

for $n, m>0$. Each homology class in $H_{n}(G, \mathbb{Z})$ is represented by an invariant cycle in $E G$. The product of two classes is given by the join of those cycles, which is an invariant cycle in $E G * E G$.

Proof We already saw that the product can be described by the join of resolutions. By the proposition above there is a degree zero chain map $C_{*}(E G) * C_{*}(E G) \rightarrow$ $C_{*}(E G * E G)$. The image of $f(1) \otimes g(1)$ under this map is the join of $f(1)$ with $g(1)$. This gives a more concrete model where the cycles are actual invariant singular cycles of the space $E G * E G$.

Corollary 3.6 The product in $\widetilde{H}_{*}(G, \mathbb{Z})$ comes from the chain map

$$
\left(P \otimes_{\mathbb{Z}[G]} \mathbb{Z}\right) \otimes_{\mathbb{Z}}\left(P \otimes_{\mathbb{Z}[G]} \mathbb{Z}\right) \rightarrow(P * P) \otimes_{\mathbb{Z}[G]} \mathbb{Z}
$$

given by $(x \otimes 1) \otimes(y \otimes 1) \rightarrow((N x) \otimes y) \otimes 1$, where $P$ is an augmented projective resolution.

This map is equal to the composition of two maps. The first one

$$
\left(P \otimes_{\mathbb{Z}[G]} \mathbb{Z}\right) \otimes_{\mathbb{Z}}\left(P \otimes_{\mathbb{Z}[G]} \mathbb{Z}\right) \rightarrow(P * P) \otimes_{\mathbb{Z}[G \times G]} \mathbb{Z}
$$

is given by $(x \otimes 1) \otimes(y \otimes 1) \rightarrow(x \otimes y) \otimes 1$. This is an exterior product, which is injective (in homology) by the Künneth theorem. Note that the homology of $(P * P) \otimes_{\mathbb{Z}[G \times G]} \mathbb{Z}$ need not be equal to $\tilde{H}_{*}(G \times G, \mathbb{Z})$ since $P * P$ is not projective over $\mathbb{Z}[G \times G]$. $(P * P) \otimes_{\mathbb{Z}[G \times G]} \mathbb{Z}$ is the chain complex of $B G * B G$, and this is the join product

$$
\widetilde{H}_{n}(B G, \mathbb{Z}) \otimes \widetilde{H}_{m}(B G, \mathbb{Z}) \rightarrow \widetilde{H}_{m+n+1}(B G * B G, \mathbb{Z}) .
$$

The second map, $(P * P) \otimes_{\mathbb{Z}[G \times G]} \mathbb{Z} \rightarrow(P * P) \otimes_{\mathbb{Z}[G]} \mathbb{Z}$ is given by $(x \otimes y) \otimes 1 \rightarrow$ $((N x) \otimes y) \otimes 1$, which is a transfer map. 


\section{Comparing Kreck's product and the cup product in Tate cohomology}

The Kreck product is defined using stratifolds and stratifold homology. Stratifolds are generalization of manifolds. They were introduced by Kreck [7] and used in order to define a bordism theory, denoted by $\mathrm{SH}_{*}$, which is naturally isomorphic to singular homology. We will use them to describe group homology with integral coefficients and the Kreck product.

\section{Stratifolds}

Kreck defined stratifolds as spaces with a sheaf of functions, called the smooth functions, fulfilling certain properties but for our purpose the following definition is enough (these stratifolds are also called p-stratifolds):

A stratifold is a pair consisting of a topological space and a subsheaf of the sheaf of real continuous functions, which is constructed inductively in a similar way to the way we construct CW-complexes. We start with a discrete set of points denoted by $X^{0}$ and define inductively the set of smooth functions which in the case of $X^{0}$ are all real functions.

Suppose $X^{k-1}$ together with a smooth set of functions is given. Let $W$ be a $n$ dimensional smooth manifold "the $n$ strata" with boundary and a collar $c$, and $f$ a continuous proper map from the boundary of $W$ to $X^{n-1}$. We require that $f$ is smooth which means that its composition with every smooth map from $X^{n-1}$ is smooth. Define $X^{n}=X^{n-1} \cup_{f} W$. The smooth maps on $X^{n}$ are defined to be those maps $g: X^{n} \rightarrow \mathbb{R}$ which are smooth when restricted to $X^{n-1}$ and to $W$ and such that for some $0<\delta$ we have $g c(x, t)=g f(x)$ for all $x \in \partial W$ and $t<\delta$.

Among the examples of stratifolds are manifolds, real and complex algebraic varieties (see Grinberg [5]), the cone over a stratifold and the product of two stratifolds.

We can also define stratifolds with boundary which are analogous to manifolds with boundary. A main difference is that every stratifold is the boundary of its cone, which is a stratifold with boundary.

Given two stratifolds with boundary $\left(T^{\prime}, S^{\prime}\right)$ and $\left(T^{\prime \prime}, S^{\prime \prime}\right)$ and an isomorphism $f: S^{\prime} \rightarrow S^{\prime \prime}$ there is a well defined stratifold structure on the space $T^{\prime} \cup_{f} T^{\prime \prime}$ which is called the gluing. On the other hand, given a smooth map $g: T \rightarrow \mathbb{R}$ such that there is a neighborhood of 0 which consists only of regular values then the preimages $g^{-1}((-\infty, 0])=T^{\prime}$ and $g^{-1}([0, \infty))=T^{\prime \prime}$ are stratifolds with boundary and $T$ is isomorphic to the gluing $T^{\prime} \cup_{\mathrm{Id}} T^{\prime \prime}$. 
To obtain singular homology we specialize our stratifolds in the following way: We use compact stratifolds, require that their top stratum will be oriented and the codimension one stratum will be empty.

Remark Regarding regularity, a condition often required, see a note by Kreck [8].

\section{Stratifold homology}

Stratifold homology was defined by Kreck in [7]. We will describe here a variant of this theory called parametrized stratifold homology, which is naturally isomorphic to it for CW-complexes. In this paper we will refer to parametrized stratifold homology just as stratifold homology and use the same notation for it.

Stratifold homology is a homology theory, denoted by $\mathrm{SH}_{*}$. It is naturally isomorphic to integral homology and gives a new geometric point of view on it.

Definition 4.1 Let $X$ be a topological space and $n \geq 0$, define $\mathrm{SH}_{n}(X)$ to be $\{g: S \rightarrow X\} / \sim$, ie, bordism classes of maps $g: S \rightarrow X$ where $S$ is a compact oriented stratifold of dimension $n$ and $g$ is a continuous map. We often denote the class $[g: S \rightarrow X]$ by $[S, g]$ or by $[S \rightarrow X] . \mathrm{SH}_{n}(X)$ has a natural structure of an Abelian group, where addition is given by disjoint union of maps and the inverse is given by reversing the orientation. If $f: X \rightarrow Y$ is a continuous map then we can define an induced map by composition $f_{*}: \mathrm{SH}_{n}(X) \rightarrow \mathrm{SH}_{n}(Y)$.

One constructs a boundary operator and prove the following:

Theorem 4.2 (Mayer-Vietoris) The following sequence is exact:

$\cdots \rightarrow \mathrm{SH}_{n}(U \cap V) \rightarrow \mathrm{SH}_{n}(U) \oplus \mathrm{SH}_{n}(V) \rightarrow \mathrm{SH}_{n}(U \cup V) \stackrel{\partial}{\rightarrow} \mathrm{SH}_{n-1}(U \cap V) \rightarrow \cdots$

where, as usual, the first map is induced by inclusions and the second is the difference of the maps induced by inclusions.

$\mathrm{SH}_{*}$ with the boundary operator is a homology theory.

Theorem 4.3 There is a natural isomorphism of homology theories $\Phi: \mathrm{SH}_{*} \rightarrow H_{*}$.

Proof See for example Tene [9]. $\Phi$ is given by $\Phi_{n}([S, f])=f_{*}([S])$ where $[S] \in$ $H_{n}(S, \mathbb{Z})$ is the fundamental class of $S$. 


\section{Stratifold group homology}

One defines the group homology of a group $G$ with coefficients in a $\mathbb{Z}[G]$ module $M$ to be $H_{*}(B G, M)$ where $M$ is considered as a local coefficients system. Our main interest is when $M=\mathbb{Z}$ with the trivial action, then this reduces to the integral homology $H_{*}(B G, \mathbb{Z})$. These groups are naturally isomorphic to the groups $\operatorname{SH}_{*}(B G, \mathbb{Z})$ by the theorem above.

Let $G$ be a compact Lie group of dimension $d$. Denote by $\operatorname{SH}_{n}(G, \mathbb{Z})$ the set of compact oriented stratifolds of dimension $n$ with a free and orientation preserving $G$ action modulo $G$-cobordism, ie a cobordism with a free $G$ action extending the given action on the boundary (all actions on the stratifolds are assumed to be smooth). We denote the class of the stratifold and the action by $[S, \rho]$.

Remark By an "orientation preserving action" we mean that the induced action of $G / G_{0}$ on $S / G_{0}$ is orientation preserving, where $G_{0}$ is the component of the identity.

The following lemma and proposition are an easy exercise:

Lemma 4.4 (1) Let $S$ be a compact oriented stratifold of dimension $n$ and $\tilde{S} \rightarrow S$ a covering space then $\widetilde{S}$ can be given a unique structure of an oriented stratifold such that the covering map is an orientation preserving local isomorphism. If $S$ is compact and the fibers are finite then $\tilde{S}$ is compact.

(2) Let $S$ be a compact oriented stratifold of dimension $n$ with an orientation preserving free action of a finite group $G$ then $S / G$ can be given a unique structure of a compact oriented stratifold such that the projection will be an orientation preserving local isomorphism.

Proposition 4.5 Let $G$ be a finite group, the map $\Psi: \operatorname{SH}_{n}(G, \mathbb{Z}) \rightarrow \operatorname{SH}_{n}(B G, \mathbb{Z})$ given be $[S, \rho] \mapsto[f: S / G \rightarrow B G]$, where $f$ is the classifying map, is an isomorphism. The map $\Psi^{-1}: \mathrm{SH}_{n}(B G, \mathbb{Z}) \rightarrow \mathrm{SH}_{n}(G, \mathbb{Z})$ is given by $[f: S \rightarrow B G] \mapsto[\tilde{S}, \rho]$ where $\widetilde{S} \rightarrow S$ is the pull back of the universal bundle $E G \rightarrow B G$ and $\rho$ is the induced action.

Remark Similarly, an isomorphism $\Psi: \mathrm{SH}_{n+d}(G, \mathbb{Z}) \rightarrow \mathrm{SH}_{n}(B G, \mathbb{Z})$ can be constructed for a compact Lie group $G$ of dimension $d$.

There is a natural product structure $\mathrm{SH}_{n}(G, \mathbb{Z}) \otimes \mathrm{SH}_{m}(G, \mathbb{Z}) \rightarrow \mathrm{SH}_{n+m}(G, \mathbb{Z})$ given by the Cartesian product with the diagonal action

$$
[S, \rho] \otimes\left[S^{\prime}, \rho^{\prime}\right] \rightarrow\left[S \times S^{\prime}, \Delta\right] .
$$


This product vanishes whenever $n, m>0$ since it is the boundary of $\left[C S \times S^{\prime}, \widetilde{\rho}\right]$ where $\tilde{\rho}$ is the obvious extension of the action $\Delta$, but it is also the boundary of $\left[S \times C S^{\prime}, \hat{\rho}\right]$ where $\hat{\rho}$ is the obvious extension of the action $\Delta$.

The Kreck product is a secondary product defined by gluing $\left(C S \times S^{\prime}, \tilde{\rho}\right)$ and $\left(S \times C S^{\prime}, \hat{\rho}\right)$ along their common boundary $\left(S \times S^{\prime}, \Delta\right)$

$$
[S, \rho] \otimes\left[S^{\prime}, \rho^{\prime}\right] \rightarrow\left[S * S^{\prime}, \rho * \rho^{\prime}\right]
$$

(note that after the gluing what we get is the join of the two stratifolds).

The product $\mathrm{SH}_{n}(G, \mathbb{Z}) \otimes \mathrm{SH}_{m}(G, \mathbb{Z}) \rightarrow \mathrm{SH}_{n+m+1}(G, \mathbb{Z})$ does not vanish in general. When $G$ is finite cyclic then $\operatorname{SH}_{n}(G, \mathbb{Z})$ is infinite cyclic when $n=0$, zero when $n$ is even and isomorphic to $G$ when $n$ is odd. The generators can be taken to be odd dimensional spheres with the action induced by the complex multiplication, when the sphere is considered as the unit sphere in a complex space. In this case the product of generators is again a generator. A similar construction will hold for $G=S^{1}$ and $S^{3}$. This implies that the product is nontrivial for every group with a free and orientation preserving smooth action on a sphere.

There is an isomorphism $\Psi: \operatorname{SH}_{n}(G, \mathbb{Z}) \rightarrow \widehat{H}^{-n-1}(G, \mathbb{Z})$ for $n>0$ given by the composition

$$
\mathrm{SH}_{n}(G, \mathbb{Z}) \rightarrow \mathrm{SH}_{n}(B G, \mathbb{Z}) \rightarrow H_{n}(B G, \mathbb{Z}) \rightarrow \hat{H}^{-n-1}(G, \mathbb{Z}) .
$$

One might show that this isomorphism is given the following way: Take some model for $E G$. Its singular chain complex $C_{*}(E G)$ is a projective resolution for $\mathbb{Z}$ over $\mathbb{Z}[G]$. Let $[(S, \rho)]$ be an element in $\operatorname{SH}_{n}(G, \mathbb{Z})$. There is a map $f: S \rightarrow E G$ that commutes with the action of $G$. This map is unique up to $G$ homotopy ( $f$ is called the classifying map), any two such maps are $G$ homotopic. Since $f$ commute with the action of $G$ it induces a map of the singular chain complexes which are complexes of $\mathbb{Z}[G]$ modules: $f_{*}: C(S) \rightarrow C(E G)$. As shown in [9], $S$ has a fundamental class, we take some representative of it which is $G$ invariant (we can do that by lifting a fundamental cycle of $S / G)$ and denote it by $s$. We get an element $f_{*}(s) \in C(E G)_{n}$ which is both invariant and a cycle thus we get an element in $\operatorname{Hom}\left(\mathbb{Z}, \Omega^{n+1}\right)$. As before different choices of $S$ and $f$ will give elements that differ by a map which factors through a projective (the fundamental class of the cobordism is mapped into $C(E G)_{n+1}$ which is projective), hence gives a homomorphism $\operatorname{SH}_{n}(G, \mathbb{Z}) \rightarrow \underline{\operatorname{Hom}}_{\mathbb{Z}[G]}\left(\mathbb{Z}, \Omega^{n+1} \mathbb{Z}\right)=\hat{H}^{-n-1}(G, \mathbb{Z})$ which is exactly the isomorphism above.

Now we would like to show that the Kreck product is the same as the cup product. We show that the join of two fundamental classes is equal to the fundamental class of their join. 
Lemma 4.6 Let $S$ and $S^{\prime}$ be two compact oriented stratifolds of dimension $n$ and $m(m, n>0)$ respectively. Denote the fundamental classes of $S, S^{\prime}, S * S^{\prime}$ by $a_{S}, a_{S^{\prime}}, a_{S * S^{\prime}}$ then $a_{S} * a_{S^{\prime}}=a_{S * S^{\prime}}$.

Proof Let $U=\left\{\left(s, s^{\prime}, t\right) \in S * S^{\prime} \mid t<1\right\}, V=\left\{\left(s, s^{\prime}, t\right) \in S * S^{\prime} \mid 0<t\right\}$ then $U \simeq$ $S, V \simeq S^{\prime}, U \cap V \simeq S \times S^{\prime}, U \cup V=S * S^{\prime}$. By Mayer-Vietoris the boundary map d: $H_{n+m+1}\left(S * S^{\prime}, \mathbb{Z}\right) \rightarrow H_{n+m}\left(S \times S^{\prime}, \mathbb{Z}\right)$ is injective (an isomorphism actually) and by the definition of the boundary we have $\partial\left(a_{S * S^{\prime}}\right)=a_{S \times S^{\prime}}$. It will be enough to show that $\partial\left(a_{S} * a_{S^{\prime}}\right)=a_{S \times S^{\prime}}$. We do know that $\partial\left(a_{S} * a_{S^{\prime}}\right)=a_{S} \times a_{S^{\prime}}$, this follows from the definition of the boundary after taking the suitable representative for $a_{S} * a_{S^{\prime}}$. So we reduced the problem to proving that $a_{S} \times a_{S^{\prime}}=a_{S \times S^{\prime}}$. This fact follows from the following commutative diagram:

$$
\begin{array}{clcc}
H_{n}(S, \mathbb{Z}) \otimes H_{m}\left(S^{\prime}, \mathbb{Z}\right) & \stackrel{\times}{\rightarrow} & H_{n+m}\left(S \times S^{\prime}, \mathbb{Z}\right) \\
\downarrow & & \downarrow \\
H_{n}(S \mid s, \mathbb{Z}) \otimes H_{m}\left(S^{\prime} \mid s^{\prime}, \mathbb{Z}\right) \stackrel{\stackrel{\times}{\rightarrow}}{\rightarrow} H_{n+m}\left(S \times S^{\prime} \mid s \times s^{\prime}, \mathbb{Z}\right)
\end{array}
$$

where $H_{k}(X \mid x, \mathbb{Z})$ stands for $H_{k}(X, X /\{x\}, \mathbb{Z})$. In order to show that $a_{S} \times a_{S^{\prime}}=$ $a_{S \times S^{\prime}}$ we have to show that for every $\left(s, s^{\prime}\right) \in S \times S^{\prime} a_{S} \times a_{S^{\prime}}$ is mapped by the right vertical map to the generator of $H_{n+m}\left(S \times S^{\prime} \mid s \times s^{\prime}\right)$. By definition $a_{S} \otimes a_{S^{\prime}}$ is mapped by the left vertical map to the tensor of the generators for $H_{n}(S \mid s, \mathbb{Z}) \otimes H_{m}\left(S^{\prime} \mid s^{\prime}, \mathbb{Z}\right)$. Also by definition the element $a_{S} \otimes a_{S^{\prime}}$ is mapped by the upper arrow to $a_{S} \times a_{S^{\prime}}$. By the commutativity of the diagram it is enough to show that the lower arrow maps the tensor of generators to the generator of the product. By excision, this can be rephrased that the same holds for the map $\times: H_{n}\left(\mathbb{R}^{n} \mid 0, \mathbb{Z}\right) \otimes H_{m}\left(\mathbb{R}^{m} \mid 0, \mathbb{Z}\right) \rightarrow$ $H_{n+m}\left(\mathbb{R}^{n+m} \mid 0, \mathbb{Z}\right)$ which is the case if we make the right choice of orientations.

We have thus proved the following theorem:

Theorem 4.7 Let $G$ be a finite group, then there is a natural isomorphism between $\mathrm{SH}_{n}(G, \mathbb{Z})$ and $\hat{H}^{-n-1}(G, \mathbb{Z})$ and this isomorphism respects the product.

In other words, the product in group homology defined by Kreck using stratifold homology and the join agrees with the cup product in negative Tate cohomology.

\section{Appendix A The stable module category}

In this appendix we give the background needed for the construction we used for Tate cohomology.

Again $R$ is a ring with unit, not necessarily commutative, and all modules are assumed to be left $R$-modules. 


\section{The stable category $\operatorname{St}-\bmod (R)$}

Let $M$ and $N$ be two $R$-modules, denote by $\operatorname{PHom}_{R}(M, N)$ the set of $R$-homomorphisms $f: M \rightarrow N$ that factors through a projective $R$-module, ie there exists a projective $R$-module $P$ and two maps $f_{1}: M \rightarrow P, f_{2}: P \rightarrow N$ such that $f=f_{2} \circ f_{1}$. The following proposition is left as an easy exercise:

Proposition A.1 $\operatorname{PHom}_{R}(M, N)$ is a submodule of $\operatorname{Hom}_{R}(M, N)$ and the composition of two homomorphisms such that one of them factors through a projective module also factors through a projective module.

By the proposition above we can define

$$
\underline{\operatorname{Hom}}_{R}(M, N)=\operatorname{Hom}_{R}(M, N) / \operatorname{PHom}_{R}(M, N)
$$

which is an $R$-module, and a composition

$$
\underline{\operatorname{Hom}}_{R}(N, K) \times \underline{\operatorname{Hom}}_{R}(M, N) \rightarrow \underline{\operatorname{Hom}}_{R}(M, K)
$$

which is $R$-bilinear.

Definition A.2 Let $R$ be a ring, denote by $\operatorname{St}-\bmod (R)$ the category whose objects are all $R$-modules and the morphisms between each $M$ and $N$ are $\underline{\operatorname{Hom}}_{R}(M, N)$. This category is called the stable module category.

\section{The functor $\Omega$}

For every $R$-module $M$ choose (once and for all) a projective cover, that is a surjective map $\pi_{M}: P_{M} \rightarrow M$ where $P_{M}$ is a projective $R$-module (for example the canonical free cover).

Define a functor $\Omega: \operatorname{St}-\bmod (R) \rightarrow \operatorname{St}-\bmod (R)$ the following way: For an object $M$ define $\Omega(M)=\operatorname{ker}\left(\pi_{M}\right)$. For a morphism $[f] \in \underline{\operatorname{Hom}}_{R}(M, N)$ choose some representative $f: M \rightarrow N$, use the fact that $P_{M}$ is projective and $\pi_{N}$ is surjective to define a map $\tilde{f}: P_{M} \rightarrow P_{N}$ such that the following diagram become commutative:

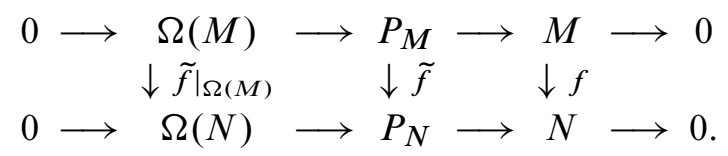

Now take $\Omega(f)$ to be the class of the induced map $\left.\tilde{f}\right|_{\Omega(f)}: \Omega(M) \rightarrow \Omega(N)$. This is well defined by the following lemma: 
Lemma A.3 (1) In the previous notation, if $\tilde{f}_{1}$ and $\tilde{f}_{2}$ are two lifts of $f \circ \pi_{M}$ then $\left.\tilde{f}_{1}\right|_{\Omega(M)}$ and $\left.\tilde{f}_{2}\right|_{\Omega(M)}$ represent the same element in $\underline{\operatorname{Hom}}_{R}(\Omega M, \Omega N)$.

(2) The map $\operatorname{Hom}_{R}(M, N) \rightarrow \underline{\operatorname{Hom}}_{R}(\Omega M, \Omega N)$ is a homomorphism.

(3) If $f$ factors through a projective then also $\left.\widetilde{f}\right|_{\Omega(f)}$ does, thus we get a homomorphism $\underline{\operatorname{Hom}}_{R}(M, N) \rightarrow \underline{\operatorname{Hom}}_{R}(\Omega M, \Omega N)$.

Proof (1) Assume we have two such lifts $\tilde{f}_{1}$ and $\tilde{f}_{2}$ then the following diagram is commutative (where $\left.h=\left.\tilde{f}_{1}\right|_{\Omega(M)}-\left.\tilde{f}_{2}\right|_{\Omega(M)}\right)$ :

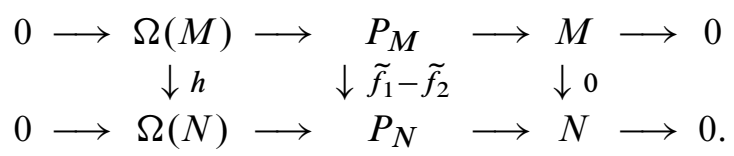

It will be enough to show that $h$ factors through $P_{M}$ which is projective. This follows from the fact that the image of the map $\widetilde{f}_{1}-\widetilde{f}_{2}$ is contained in $\Omega(N)$ by the commutativity of the diagram.

(2) Choose the lifting of $a \cdot f+b \cdot g$ to be $a \cdot \tilde{f}+b \cdot \widetilde{g}$.

(3) Assume $f$ factors through a projective module $P$. We have the following diagram:

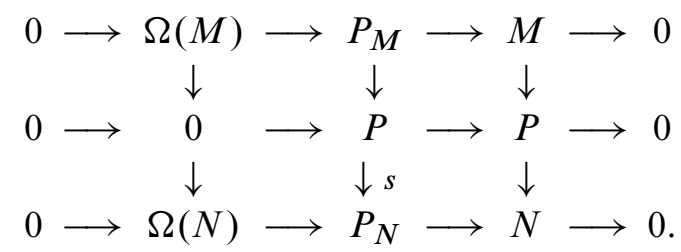

The map $s: P \rightarrow P_{N}$ can be defined using the fact that $P$ is projective and the map $P_{N} \rightarrow N$ is surjective. Thus the induced map $\Omega(M) \rightarrow \Omega(N)$ is the zero map.

The following is important for the definition of Tate cohomology:

Proposition A.4 Let $G$ be a finite group and $R=\mathbb{Z}[G]$. If $M$ is a $\mathbb{Z}[G]$ module which is projective as an Abelian group then the map $\underline{\operatorname{Hom}}_{R}(M, N) \rightarrow \underline{\operatorname{Hom}}_{R}(\Omega M, \Omega N)$ is an isomorphism.

Proof Before we start recall [2, VI 2] that a $\mathbb{Z}[G]$-module $Q$ is called relatively injective if for every injection $A \hookrightarrow B$ of $\mathbb{Z}[G]$-modules which splits as an injection of Abelian groups and every $\mathbb{Z}[G]$ homomorphism $A \rightarrow Q$ there exists an extension to a $\mathbb{Z}[G]$ homomorphism $B \rightarrow Q$, and that if $G$ is a finite group every projective module is relatively injective. 
We construct an inverse to this map. Given a map $f: \Omega M \rightarrow \Omega N$. We have the following diagram:

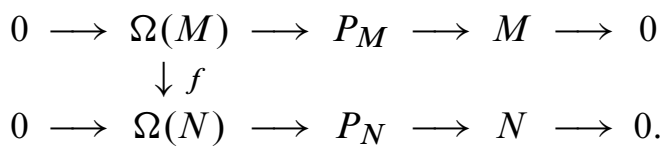

Since $M$ is projective as an Abelian group the upper row splits as Abelian groups. This means that $\Omega(M) \longrightarrow P_{M}$ is a split injection as Abelian groups. $P_{N}$ is projective and hence relatively injective therefore we can extend the homomorphism $\Omega(M) \rightarrow P_{N}$ to a homomorphism $\tilde{f}: P_{M} \rightarrow P_{N}$ such that the diagram will commute. This induces a homomorphism $\bar{f}: M \rightarrow N$. Of course $\bar{f}$ depends on the choice of $\tilde{f}$. Suppose that $\widetilde{f}_{1}, \widetilde{f}_{2}$ are two extensions then $\widetilde{f}_{1}-\widetilde{f}_{2}$ vanishes on $\Omega(M)$ hence the map $\overline{f_{1}}-\overline{f_{2}}: M \rightarrow N$ factors through $P_{N}$ which is projective. This gives a well defined homomorphism $\operatorname{Hom}_{R}(\Omega M, \Omega N) \rightarrow \underline{\operatorname{Hom}}_{R}(M, N)$. Assume $f: \Omega M \rightarrow \Omega N$ factors through a projective $P$ then we can choose $\tilde{f}$ to factor through $P$ again since it is relatively injective and get that $\bar{f}$ is the zero map:

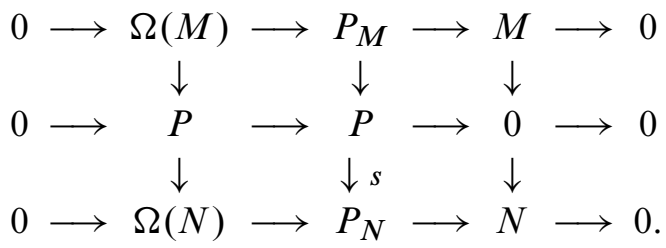

Hence we get a homomorphism $\underline{\operatorname{Hom}}_{R}(\Omega M, \Omega N) \rightarrow \underline{\operatorname{Hom}}_{R}(M, N)$ which is easily seen to be the inverse of the homomorphism $\underline{\operatorname{Hom}}_{R}(M, N) \rightarrow \underline{\operatorname{Hom}}_{R}(\Omega M, \Omega N)$.

We have defined the endofunctor $\Omega$. We define $\Omega^{n}$ by induction: $\Omega^{0}=\operatorname{Id}$ and $\Omega^{n}=\Omega \circ \Omega^{n-1}$.

Proposition A.5 Let $M$ be an $R$-module and let $\cdots \rightarrow Q_{n-1} \rightarrow \cdots \rightarrow Q_{0} \rightarrow M$ be any projective resolution of $M$, then $\Omega^{n}(M)$ can be identified with $\operatorname{ker}\left(Q_{n-1} \rightarrow Q_{n-2}\right)$, that is there is a canonical map $\operatorname{ker}\left(Q_{n-1} \rightarrow Q_{n-2}\right) \rightarrow \Omega^{n}(M)$ which is an isomorphism in the category $\mathrm{St}-\bmod (R)$.

Proof Given an $R$-module $M$ we construct a canonical projective resolution of it using the projective covers we have chosen before. We do it by induction where $P_{n}$ is defined to be the projective cover of $\operatorname{ker}\left(P_{n-1} \rightarrow P_{n-2}\right)$ with the induced map $P_{n} \rightarrow P_{n-1}$, which clearly make this into a projective resolution. Notice that by the definition of $\Omega$ we have $\Omega^{n}(M)=\operatorname{ker}\left(P_{n-1} \rightarrow P_{n-2}\right)$, and for a map $f: M \rightarrow N$ the map $\Omega^{n}(f)$ can be constructed by extending the map $f$ to a chain map between the 
two resolutions. In order to prove the proposition it will suffice to show that given two projective resolutions of $M \cdots \rightarrow Q_{n-1} \rightarrow \cdots \rightarrow Q_{0} \rightarrow M$ and $\cdots \rightarrow P_{n-1} \rightarrow \cdots \rightarrow$ $P_{0} \rightarrow M$ there is a canonical isomorphism $\operatorname{ker}\left(Q_{n-1} \rightarrow Q_{n-2}\right) \rightarrow \operatorname{ker}\left(P_{n-1} \rightarrow P_{n-2}\right)$. This follows directly by induction from what we have already showed in the case of a the projective cover of $M$.

Remark By similar reasons we can compute the induced maps $\Omega^{n}(f)$ for any map $f: M \rightarrow N$ by taking any two resolutions for $M$ and for $N$ and extending $f$ into a chain map between the two resolutions.

\section{References}

[1] D J Benson, J F Carlson, Products in negative cohomology, J. Pure Appl. Algebra 82 (1992) 107-129 MR1182934

[2] K S Brown, Cohomology of groups, Graduate Texts in Math. 87, Springer, New York (1982) MR672956

[3] J F Carlson, L Townsley, L Valeri-Elizondo, M Zhang, Cohomology rings of finite groups, Algebras and Appl. 3, Kluwer, Dordrecht (2003) MR2028960 With an appendix: Calculations of cohomology rings of groups of order dividing 64 by Carlson, Valeri-Elizondo and Zhang

[4] G Fors, On the foundation of algebraic topology arXiv:math/0412552

[5] A Grinberg, Resolution of stratifolds and connection to Mather's abstract prestratified spaces, PhD thesis, Ruprecht-Karls-Universität Heidelberg (2003) Available at http://archiv.ub.uni-heidelberg.de/volltextserver/volltexte/ 2003/3127/pdf/gri\% nberg_diss.pdf

[6] M Kreck, Equivariant stratifold cohomology, equivariant Poincaré duality and equivariant characteristic classes, preprint

[7] M Kreck, Differential algebraic topology: From stratifolds to exotic spheres, Graduate Studies in Math. 110, Amer. Math. Soc. (2010) MR2641092

[8] M Kreck, Pseudo homology, p-stratifold homology and ordinary homology, preprint (2011)

[9] H Tene, Stratifolds and equivariant cohomology theories, $\mathrm{PhD}$ thesis, University of Bonn (2010) Available at http://hss.ulb.uni-bonn.de/2011/2391/2391.pdf

Department of Mathematics and PMI, POSTECH

Pohang 790-784, South Korea

teneh@postech.ac.kr

http://math.postech.ac.kr/ teneh

Received: 3 April $2011 \quad$ Revised: 24 November 2011 